\title{
TESTING OF THE NEW TUNER DESIGN FOR THE CEBAF 12 GEV UPGRADE SRF CAVITIES*
}

\author{
E. F. Daly", G. K. Davis and W. R. Hicks \\ Thomas Jefferson National Accelerator Facility, Newport News, VA 23606
}

\begin{abstract}
The new tuner design for the $12 \mathrm{GeV}$ Upgrade SRF cavities consists of a coarse mechanical tuner and a fine piezoelectric tuner. The mechanism provides a 30:1 mechanical advantage, is pre-loaded at room temperature and tunes the cavities in tension only. All of the components are located in the insulating vacuum space and attached to the helium vessel, including the motor, harmonic drive and piezoelectric actuators. The requirements and detailed design are presented. Measurements of range and resolution of the coarse tuner are presented and discussed.
\end{abstract}

\section{INTRODUCTION}

The third in a series of three cryomodules $(\mathrm{CMs})$ is nearly complete as part of JLab's efforts to produce prototypical CMs for the $12 \mathrm{GeV}$ Upgrade of CEBAF [1]. This CM, dubbed Renascence, incorporates several design changes in order to provide more than the $108 \mathrm{MV}$ capability required by present plans for the $12 \mathrm{GeV}$ upgrade [2]. One particular design change is an invacuum, all-cold cavity frequency tuner, capable of tuning the upgrade cavities (Table 1).

Table 1: Pertinent Cavity Information

\begin{tabular}{|l|c|}
\hline Parameter & Value \\
\hline \hline Frequency $(\mathrm{MHz})$ & 1497 \\
\hline Gradient $(\mathrm{MV} / \mathrm{m})$ & 19.2 \\
\hline Operating Mode & $\mathrm{CW}$ \\
\hline Qexternal & $2 \times 10^{7}$ \\
\hline Bandwidth $(\mathrm{Hz})$ & $\sim 75$ \\
\hline Frequency Sensitivity $(\mathrm{Hz} / \mu \mathrm{m})$ & $\sim 300$ \\
\hline $\begin{array}{l}\text { Lorentz Detuning at Full } \\
\text { Gradient }(\mathrm{Hz})\end{array}$ & $\sim 500 \mathrm{~Hz}$ \\
\hline Spring Constant $(\mathrm{kN} / \mathrm{mm})$ & $5.6-8.4$ \\
\hline
\end{tabular}

\section{TUNER REQUIREMENTS}

The tuner is similar to the version designed for the first prototype CMs since it consists of a coarse mechanical tuner and a fine piezoelectric tuner, and satisfies the same design requirements (Table 2). The current design approach is identical to the SNS tuner and relies on component evaluation tests conducted previously to prove out the SNS tuner system. Based on these tests, it is expected that the upgrade tuner system will meet or exceed its design requirements.

\footnotetext{
* Supported by US DOE Contract No. DE-AC05-84ER40150 "eddaly@jlab.org
}

The new cavity design requires two additional higherorder mode (HOM) couplers. The couplers are located closer to the cavity end cells to increase the coupling [3]. This new configuration prevents the tuner mechanism from occupying beam line space. Therefore, the new tuner is mounted on the helium vessel shell with the tuning force transmitted through the helium vessel heads to the cavities.

It is desirable to have systems that maximize accelerator runtime. Therefore, components should be highly reliable, maintainable and repairable. The cyclic life requirement for the coarse tuner is based on tuning twice per day, every day for forty years. The cyclic life requirement for the fine tuner is based on turning on or tuning twenty times per hour, all day, every day for forty years. Based on accelerated life test results for the SNS tuner mechanism, the requirements have been satisfied for the coarse and fine tuners.

The vacuum and radiation requirements are consistent with the original CEBAF CM and dictate the use of dry lubricants within the moving components.

Table 2: Tuner Requirements

\begin{tabular}{|l|c|}
\hline Parameter & Value \\
\hline \hline Coarse Range (kHz) & $>400$ \\
\hline Coarse Resolution (Hz) & $<100$ \\
\hline Backlash (Hz) & $<25$ \\
\hline Fine Range (Hz) & 1000 \\
\hline Fine Resolution (Hz) & $<1$ \\
\hline Cyclic Life - Coarse Tuner cycles) & $29 \times 10^{3}$ \\
\hline Cyclic Life - Fine Tuner (cycles) & $7 \times 10^{6}$ \\
\hline Temperature Range (K) & $2-300$ \\
\hline Vacuum Environment (torr) & $10^{-6}$ \\
\hline Radiation Limit (rads) & $>10^{6}$ \\
\hline
\end{tabular}

\section{DESIGN DESCRIPTION}

The tuner consists of several components - the mechanical linkage, the gear reduction, the stepper motor and two piezoelectric actuators (Figure 1). All of the components are in vacuum and cold. The $316 \mathrm{~L}$ stainless steel mechanism provides a 30:1 mechanical advantage and is pre-loaded at room temperature. The cavities when cold are tuned in tension only by the actuation of a stepper motor that provides 200 steps per revolution. Clockwise rotation of the motor, when looking at the end opposite the output shaft, results in an increase in cavity frequency. The motor [4] can deliver a torque up to 0.37 $\mathrm{N} * \mathrm{~m}$ (52 in*oz). The harmonic drive [5] provides a gear reduction of 100:1 between the motor and drive screw. 


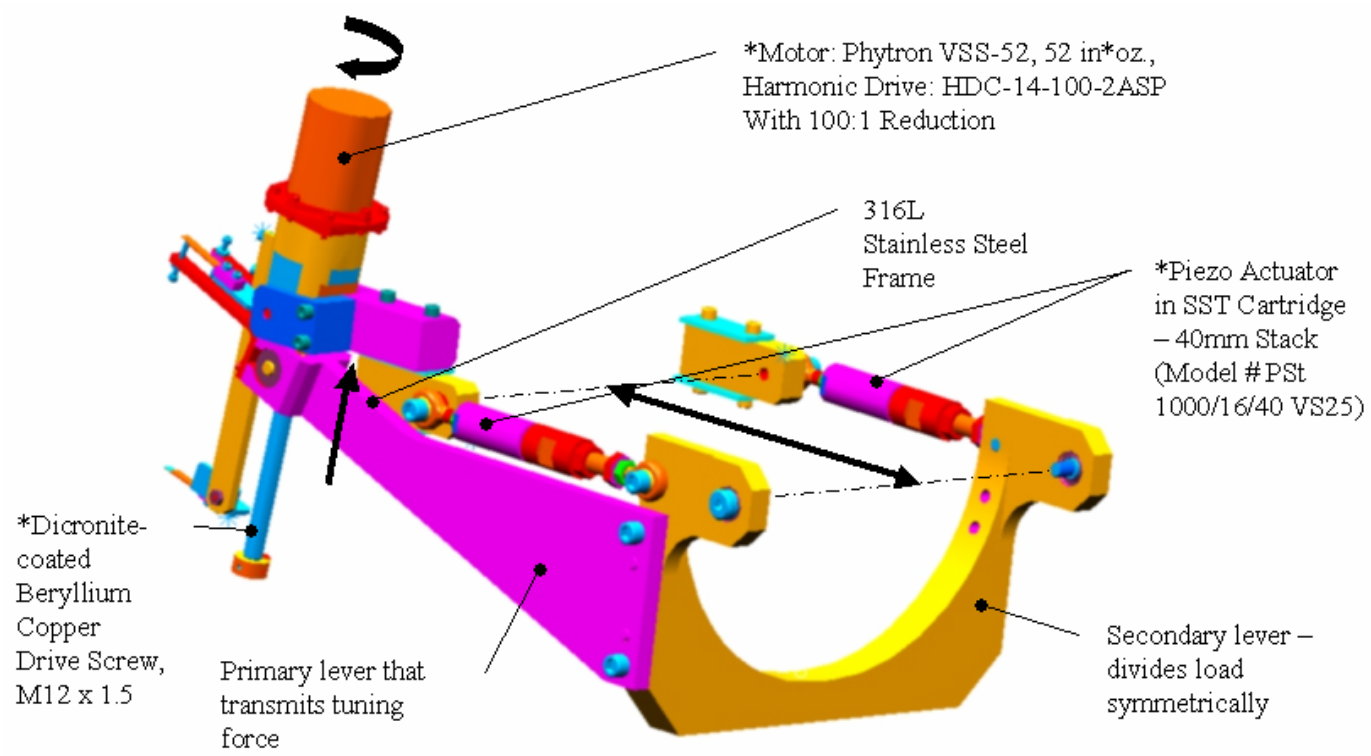

Figure 1. Upgrade Tuner Mechanism

The drive screw material is $\mathrm{BeCu}$, chosen for its strength and hardness. The drive screw and all bearing components are coated with Dicronite ${ }^{\mathrm{TM}}$. The drive screw moves the primary lever relative to the motor.

Force and displacement are distributed evenly via the secondary lever to a pair of struts. The struts, acting in compression, rotate through an angle of $6^{\circ}$ about the end closest to the motor and push on the helium vessel shell and head (not shown) to stretch the cavity in tension. This portion of the mechanism can apply a large force over a relatively short distance.

It is important to note that the structural requirements for the helium vessel head and shell are more stringent than for previous designs since the cavity tuning forces are transmitted through these components. Additional reinforcing has been added to the helium vessel heads to limit the distortion under tuning loads.

Two high-voltage piezoelectric actuators [6], a primary and a secondary, are loaded compressively during tuning and provide fine tuning capabilities that can be used for fast turn-on or possibly even microphonics control.

The tuner motor and harmonic drive, key components of the tuning system, must be accessed for repair or replacement if necessary. A flanged port on the vacuum tank provides access and also contains electrical feedthroughs for stepper motor power, limit switch signals and piezoelectric actuator power. One of the two piezoelectric actuators can be removed and replaced through this port in the event of failure of both the primary and secondary actuators.

\section{TEST SET-UP}

Two cavities were tested - the high-gradient prototype (HGPT) and a low-loss (LL) cavity, serial number LL004. The cavity, helium vessel and tuner assemblies were suspended on a top plate suitable for installation into a dewar in the Vertical Test Area (VTA), as done in a previous test of the prototype tuner assembly attached to a test cavity, serial number EP3 [7]. Low power measurements were taken using a one-watt amplifier to supply power to the cavity and a network analyzer to measure the pi-mode frequency of the cavity.

A National Instruments NI-7344 stepper motor controller [8] was used to power the tuner motor during the tests. It was configured to provide two micro-steps per full motor step, commonly called half-stepping. The device can be configured to provide up to 256 micro-steps per step, which can provide very fine stepping resolution. However, when set for very fine micro-stepping levels, some motors can exhibit a reduction in output torque.

\section{RESULTS}

The relationship between the stepper motor steps and cavity response is monotonically increasing as predicted by calculations (Figure 2). The two sets of data are linear in the range between zero and $400 \mathrm{kHz}$. A linear curve fit of the data in this range yields a tuning sensitivity of $0.73 \mathrm{~Hz}$ per step for the HGPT cavity and $0.57 \mathrm{~Hz}$ per step for the LL004 cavity. The $\mathrm{R}^{2}$ value for both of these curve fits was 0.99 . Tuning sensitivities, still very linear over the larger range of zero to $800 \mathrm{kHz}$, were 0.66 and $0.51 \mathrm{~Hz}$ per step for the HGPT and LL004 cavities respectively.

The tuner range is approximately $1 \mathrm{MHz}$, exceeding the requirement by a factor of 2.5. The decrease in frequency change is expected as the number of steps increases since the tuning mechanism is analytically described as a cosine function. For very small angles of the struts (as shown in Figure 1), the actual change in length of the cavity approaches zero.

The tuner resolution is measured by driving the motor one full step increments every five seconds, recording the frequency change as a function of time and then 


\section{ACKNOWLEDGEMENTS}

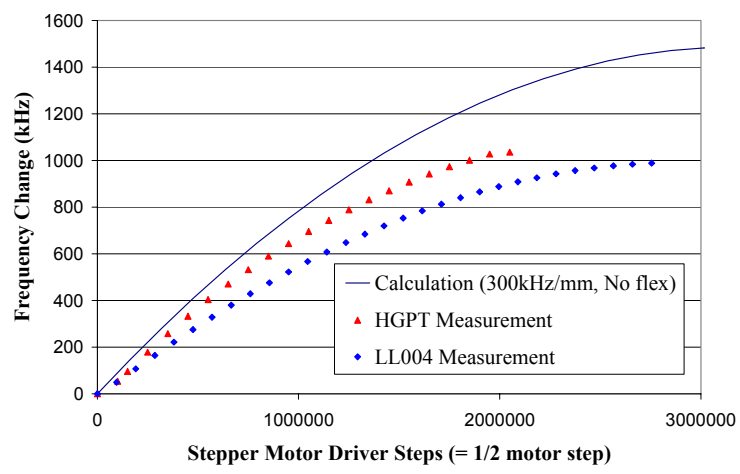

Figure 2. Coarse Tuner Range

measuring the peak-to-peak frequency change (Figure 3). The resolution, measured on the EP3 test cavity is $1.7 \mathrm{~Hz}$.

For smooth integration into the CEBAF control system, it is important to know how the upgrade cavity frequency changes with stepper motor actuation. To know this, the stepper motor revolution direction must be defined. For the original CEBAF CMs, the clockwise direction is defined when looking at the back of the motor towards the cavity and mechanism. Using this convention, the cavity frequency increases as the motor is rotated clockwise.

\section{CONCLUSION}

Results from testing have shown that the new tuner design meets requirements and that the coarse tuner range is sufficient for the $12 \mathrm{GeV}$ Upgrade CM. The coarse tuner range exceeds $1 \mathrm{MHz}$ and the tuning sensitivities are less than $1 \mathrm{~Hz}$ per step.

\section{FUTURE WORK}

Further testing, planned for the summer of 2005, will include verification of range and resolution for the coarse and fine tuners in the Renascence CM in the Cryomodule Test Facility (CTF).

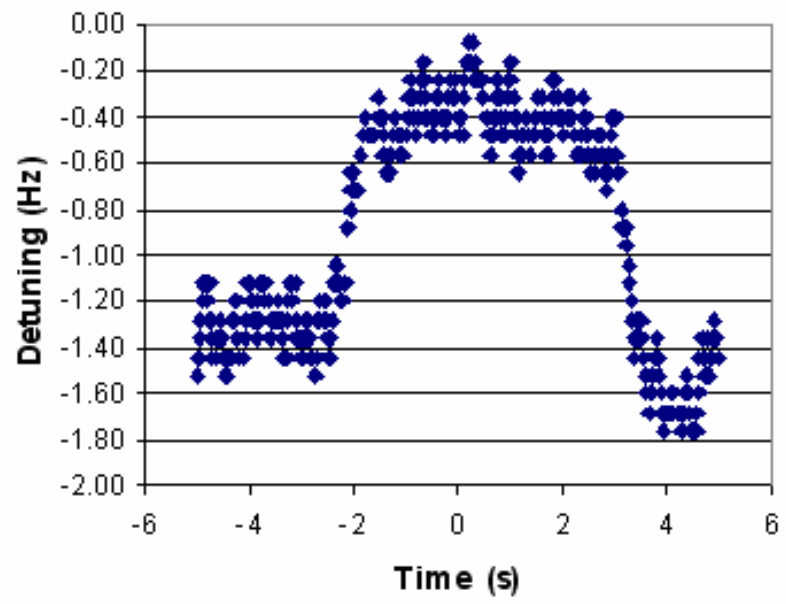

Figure 2. Coarse Tuner Range
The authors would like to thank Kurt Macha, Pam Morrison, and Pete Kushnick of JLab and Terry Penisi of SNS-ORNL for their assistance with the test apparatus.

\section{REFERENCES}

[1] E. F. Daly et al., "Improved Prototype Cryomodule for the CEBAF $12 \mathrm{GeV}$ Upgrade," Proc. PAC2003, p. 1377.

[2] K. M. Wilson et al., "Mechanical Cavity Design for 100MV Upgrade Cryomodule," Proc. PAC2003, p. 2866.

[3] C. E. Reece et al., "Fabrication and Testing of the SRF cavities for the CEBAF $12 \mathrm{GeV}$ Upgrade Prototype Cryomodule Renascence," published in these proceedings.

[4] Motor information - www.phytron.com

[5] Harmonic drive information - www.harmonicdrive.com

[6] Piezoelectric actuator information www.piezomechanik.com

[7] R. Hicks et al., "Analysis and Testing of the Prototype Coarse Tuner for the Renascence Cryomodule," JLab Tech. Note TN-04-003.

[8] NI Stepper motor controller - www.ni.com 